\title{
Reducing Extra-Terrestrial
}

\section{Excavation Forces with Percussion}

\author{
Robert Mueller \\ NASA, Kennedy Space Center \\ Mail Stop: NE-S \\ Kennedy Space Center, FL \\ 32899 \\ 321-867-2557 \\ Rob.Mueller@nasa.gov \\ Jason Schuler \\ ESC, Kennedy Space Center \\ Mail Stop: ESC-58 \\ Kennedy Space Center, FL \\ 32899 \\ 321-867-7854 \\ Jason.M.Schuler@nasa.gov
}

\author{
Jonathan Drew Smith \\ NASA, Kennedy Space Center \\ Mail Stop: NE-S \\ Kennedy Space Center, FL \\ 32899 \\ 321-867-8726 \\ Jonathan.D.Smith@nasa.gov
}

\author{
Andrew Nick \\ ESC, Kennedy Space Center \\ Mail Stop: ESC-58 \\ Kennedy Space Center, FL \\ 32899 \\ 321-867-4873 \\ Andrew.J.Nick@nasa.gov
}

\author{
Thomas Lippitt \\ NASA, Kennedy Space Center \\ Mail Stop: NE-S \\ Kennedy Space Center, FL \\ 32899 \\ 321-867-1391 \\ Thomas.Lippitt@nasa.gov
}

\begin{abstract}
High launch costs and mission requirements drive the need for low mass excavators with mobility platforms, which in turn have little traction and excavation reaction capacity in low gravity environments. This presents the need for precursor and long term future missions with low mass robotic mining technology to perform In-Situ Resource Utilization (ISRU) tasks. This paper discusses a series of experiments that investigate the effectiveness of a percussive digging device to reduce excavation loads and thereby the mass of the excavator itself. A percussive mechanism and 30" wide pivoting bucket were attached at the end of the arm simulating a basic backhoe with a percussion direction tangent to the direction of movement. Impact energies from $13.6 \mathrm{~J}$ to $30.5 \mathrm{~J}$ and frequencies from 0 BPM to 700 BPM were investigated. A reduction in excavation force of as much as $\mathbf{5 0 \%}$ was achieved in this experimental investigation.
\end{abstract}

\section{TABLE OF CONTENTS}

1. INTRODUCTION .1

2. EXPERIMENTAL SETUP.......................................2

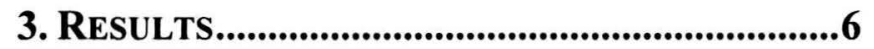

4. CONCLUSIONS .......................................................9

5. ACKNOWLEDGEMENTS....................................10

REFERENCES......................................................10

BIOGRAPHY ........................................................11

\section{INTRODUCTION}

\section{The Need for Reduced Excavation Forces}

Outer Space contains a vast amount of resources in the form of energy and materials. The energy is in the form of solar, thermal, magnetic and other sources, but this paper is concerned with acquiring accessible materials which are mostly contained in the layers of planetary surface ejecta debris known as regolith. The regolith contains compounds of metals and minerals which can be processed to extract useful materials such as oxygen, hydrogen, iron, aluminum, titanium, water, carbon and more. However, Lunar missions, Mars missions, and Near Earth Objects (NEO) missions have all shown that many planetary bodies only have loose unconsolidated regolith in the first few centimeters of depth below the surface. After that, the regolith becomes compacted and consolidated due to billions of years of vibration and thermal cycling [Schmitt et al, 1991]. In addition, hydrogen signatures and water ice measurements in induced ejecta plumes [Colaprete et al, 2010] show that there is a high probability of frozen icy regolith (containing water) being present at the Lunar poles. Other planets such as Mars also have water ice present at the polar locations as evidenced by the NASA Mars Phoenix Mission [Smith et al, 2009].

The first step towards utilization of the regolith resources on an extra-terrestrial body is acquiring or manipulating the regolith as a feedstock for some process. Examples of surface systems tasks that have been identified which require the use of regolith excavation are [Mueller, 2006]:

1. Surface Preparation and Construction of a Landing and Launch Area

2. Deployment of a Sub-surface Power Plant (Shielded by burying it)

3. Shielding of Human Living and Working Spaces

4. The Search for Extra-Terrestrial Water

5. Regolith Excavation for In-Situ Resource Utilization (ISRU) $\mathrm{O} 2$ and $\mathrm{H} 2$ Production

6. Sub-Surface Mobility for Exploration and Science

7. Mining for Commercial Export of Resources (Platinum, $\mathrm{H}_{2} \mathrm{O}$, Helium 3 et.c.)

These tasks all imply excavation of some type. The 
inherent challenge in excavating the consolidated regolith is that it becomes very dense with bulk densities having been measured on the Earth's moon of up to $1.8 \mathrm{grams} /$ cubic centimeter [Schmitt et al, 1991]. The addition of water ice means that the regolith may have an even higher bulk density, and if it is not in a permanently shadowed region, then the water ice can only be stable if it is insulated by an over burden of regolith, which must be removed to access it. On Earth a construction excavation machine typically uses a brute force approach to excavate soil and other granular materials. Conversely, when operating in the reduced gravity environments of the moon, Mars and NEO's, there is insufficient reaction force and traction to counteract the digging forces from the excavation implement, so novel ways of excavating and transporting regolith must be found. On Earth other methods such as pneumatic transfer of granular materials, and excavation using explosives has been used, but due to the unique vacuum environments and low gravities, such methods can be impractical. New methods must be found for extra-terrestrial excavation of regolith in these very different environments.

\section{Methods of Reducing Excavation Forces}

The Granular Mechanics \& Regolith Operations (GMRO) laboratory operates at the NASA, Kennedy Space Center (KSC) Swamp Works: a facility which is managed by the KSC Surface Systems Office. The GMRO lab is dedicated to researching and developing the critical regolith technologies necessary for human and robotic endeavors throughout the Solar System. As such, there is a high degree of interest in developing methods and means for the reduction or mitigation of excavation forces in highly consolidated regoliths on various extra-terrestrial bodies. Since launch mass and volume are highly constrained due to the high costs involved, it is desirable and appropriate that smaller and more lightweight excavation machines should be developed to enable regolith resource utilization in space.

Several methods have been investigated in order to reduce or mitigate the excavation forces in regolith. Among these are; pneumatic excavation, percussive excavation, counter-rotating excavation bucket drums, scraping systems, brush systems, augers, bucket ladders, bucket wheels, draglines, and overshot loaders. In addition, NASA has collaborated with innovative small commercial companies such as Astrobotic Technology, Inc. to investigate the "Parameters Governing Regolith Site Work by Small Robots [Skonieczny, K et al, 2010] and Honeybee Robotics, inc. to develop a "Five-Step Parametric Prediction and Optimization Tool for Lunar Surface Systems Excavation Tasks" [Zacny. $\mathrm{K}$ et al, 2010]. This paper is focused on the experimental and empirical investigation of a specific implementation of a percussive excavation bucket style implement that was custom designed to be retro-fitted to the existing All-Terrain Hex-Limbed Extra-Terrestrial Explorer (ATHLETE) vehicle developed by NASA at the Jet Propulsion Lab (JPL), [Wilcox et al, 2007].

\section{Previous Percussive Excavators}

Percussive excavation has been used in industry for many years in the form of drills and jack hammers. This implementation requires a minimum of two tools to fully break apart the rock and extract the material. Previous to this project there have been efforts to try and combine the two extraction methods into one implement. In recent years, Honeybee Robotics has studied this new technique in percussive excavation. Honeybee Robotics performed tests with a Lunar Surveyor sized scoop, $5.08 \mathrm{~cm}$ (2 in). Their test setup plowed the surveyor bucket through soil and varied percussion frequency and impact energy to determine the effect on excavation load [Reference Honeybee paper]. Testing with a $10 \mathrm{~cm}$ wide trenching bucket was also conducted under the NASA Kennedy Space Center (KSC) Undergraduate Student Research Program (USRP) in 2008. A trenching bucket was pulled through JSC-1A while a voice-coil actuator vibrated the cutting edge. [Reference Matt Whittaker Paper]. While the KSC USRP trenching experiment was somewhat inconclusive the Honeybee Robotics experiment proved that this technology is a viable option for reducing excavation loads.

\section{EXPERIMENTAL SETUP}

\section{Viper Mechanism}

Vibratory Impacting Percussive Excavator for Regolith (VIPER) is a percussive backhoe style bucket designed to attach to ATHLETE. At the end of each ATHLETE limb a quick connect interface allows various tools to be attached. VIPER attaches to ATHLETE via this interface and the percussive mechanism is driven from a power take-off on the ATHLETE wheel motor. This interface is purely mechanical with no electrical connections.

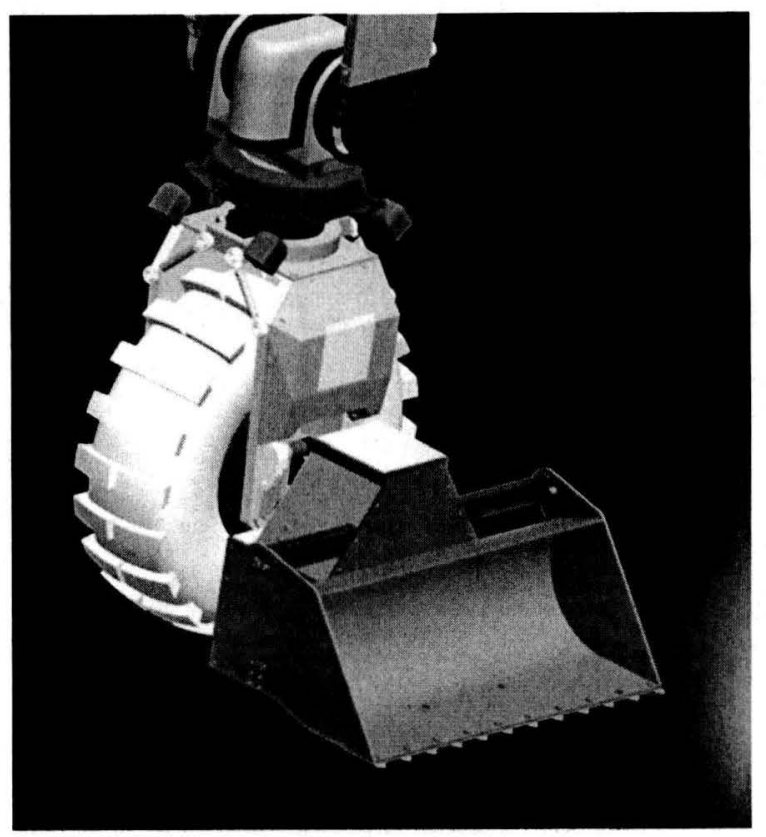

Figure 1 - VIPER on ATHLETE 
VIPER was designed to dig up to 1 meter deep and trench in a range of regolith densities. The bucket is $76 \mathrm{~cm}$ (30 in) wide and can hold a volume of $35000 \mathrm{~cm}^{3}\left(2140 \mathrm{in}^{3}\right)$. The minimum width of the bucket was equal to the diameter of the wheel on ATHLETE to allow VIPER to trench (see figure 1).

To be able to test different variables such as frequency and impact energy independently a barrel cam (see figure 2) was used to compress a die spring then release the spring and drive a hammer into the anvil which is attached to the bucket. The anvil is in line with cutting edge of the bucket to directly transfer the impact energy to the simulant and the bucket is free to rotate about a pivot. Using this style of mechanism to achieve the percussion, the impact energy could be varied by replacing the die springs and the frequency could be varied by changing the RPM of the motor. This mechanism was chosen over an electrical jackhammer crank-piston mechanism due to its lack of variability. The crank-piston mechanism's frequency and impact energy are tied to the RPM of the motor, therefore not allowing independent variables without changing parts for every test.

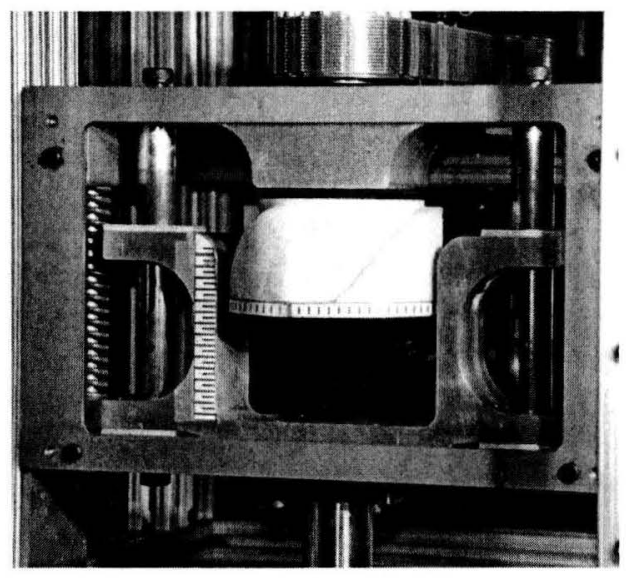

Figure 2 - Testing of Barrel Cam

Honeybee Robotics also used a barrel cam mechanism in their testing. This mechanism may not be the most efficient for an optimized design if a specific frequency and impact energy were determined to be effective; however for an experimental setup it is appropriate. The VIPER mechanism was designed to provide impact energies of $13.6 \mathrm{~J}, 19.3 \mathrm{~J}$, $30.5 \mathrm{~J}$ and a percussive frequency range of $0-900$ beats per minute (BPM). These ranges were chosen based upon the Honeybee Robotics data which showed about a $70 \%$ reduction using a $2.5 \mathrm{~J}$ spring at $1000 \mathrm{BPM}$.

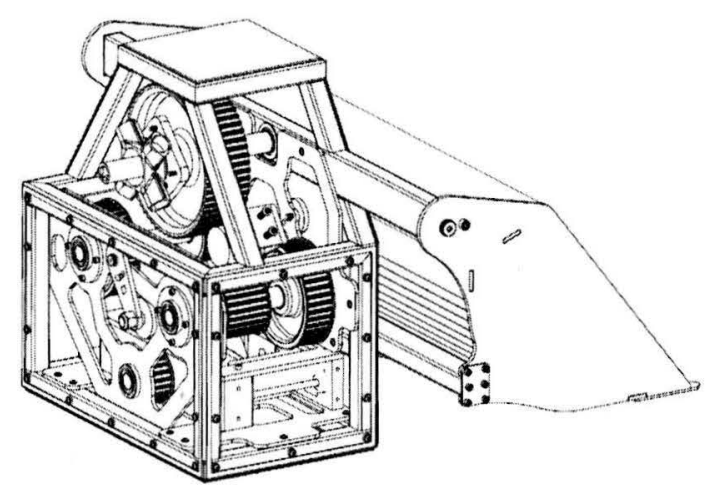

Figure 3 - VIPER with gearing shown

To define a range of impact energies and frequencies for the VIPER mechanism it was assumed that frequency did not scale with bucket width and impact energy scaled linearly with bucket width. The linear scaling of the impact energy was based upon the Luth and Wismer plowing model for horizontal force in cohesionless sand that showed that horizontal excavation force increases linearly with bucket width [Luth and Wismer]. Frequency was assumed not to scale based upon the hypothesis that if enough impact energy was supplied to disrupt the simulant then the frequency would only need to be high enough to keep the simulant fluid.

\section{Test stand and DAQ System}

The Viper Test Stand (VTS) is a simple truss structure with a pivoting arm that allows the bucket to excavate through an arcing path. This path was assumed to be a good analog for a backhoe excavation profile. The depth of cut is adjusted on the pivoting arm by a motor and acme screw assembly and the rotation of the arm is driven by a winch attached to the base structure. Both depth and angle were measured using string potentiometers. A load cell was placed in line with the winch cable to record the load during testing. The excavation cutting edge load was then resolved from the load cell value into a total resultant excavation force that was assumed to be tangential to the arc around the pivot (see figure 4). Frequency was controlled through a motor controller with a PI control loop and was not actively measured during testing, although during bench-top testing a high speed camera was used to verify the motor controller maintained commanded frequency and post processing of the test data reflects the commanded frequency of percussion in the raw load cell data. Impact energy was not measured and was recorded as a spring potential energy. Excavation speed was found using the measured angle over time. A LabVIEW DAQ system recorded the angle, depth, time, and load at $500 \mathrm{~Hz}$ during testing. 
Note: Cut depth is also recorded through string pot.

\section{VIPER Test Stand (VTS) FBD}

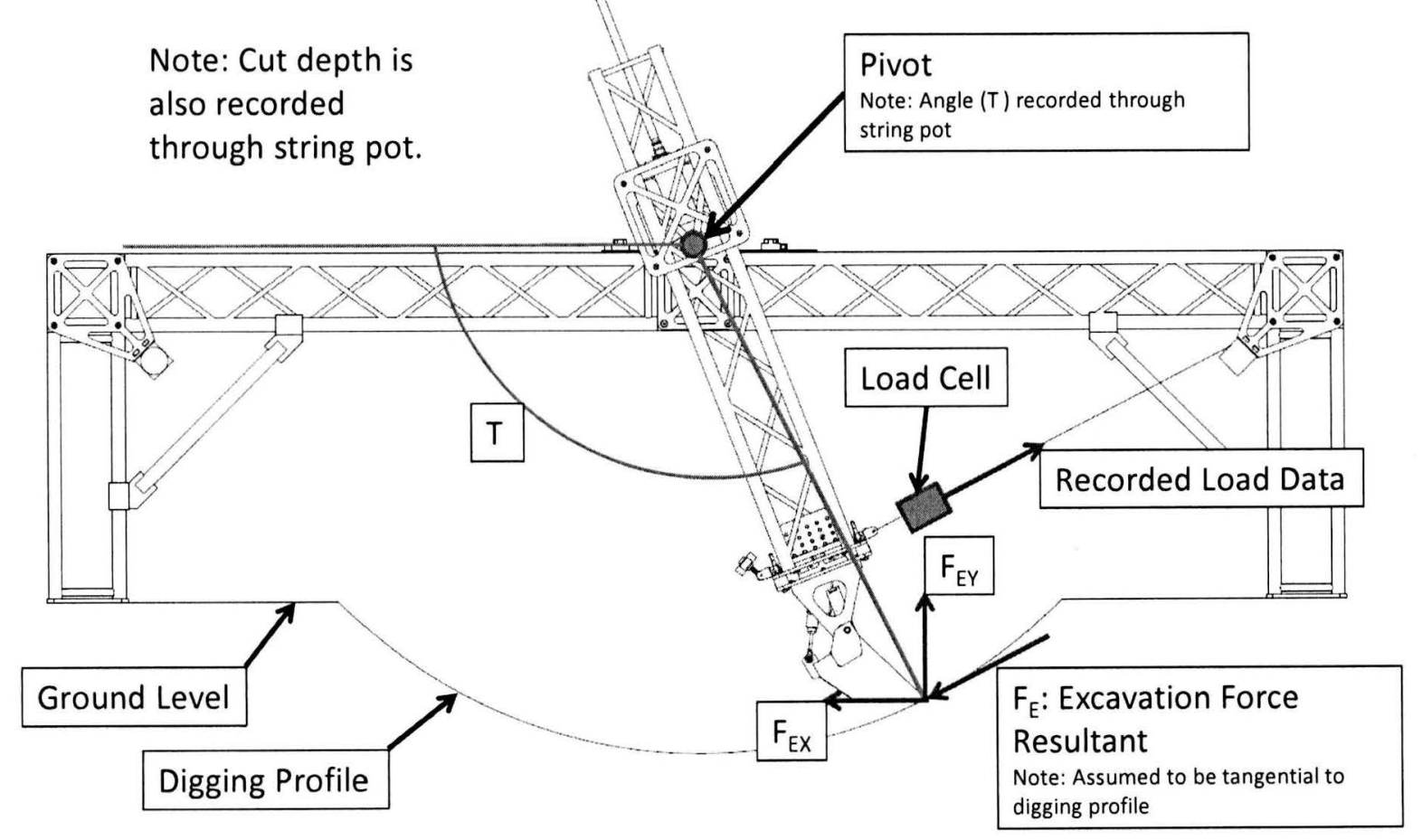

Figure 4 - VTS Free Body Diagram

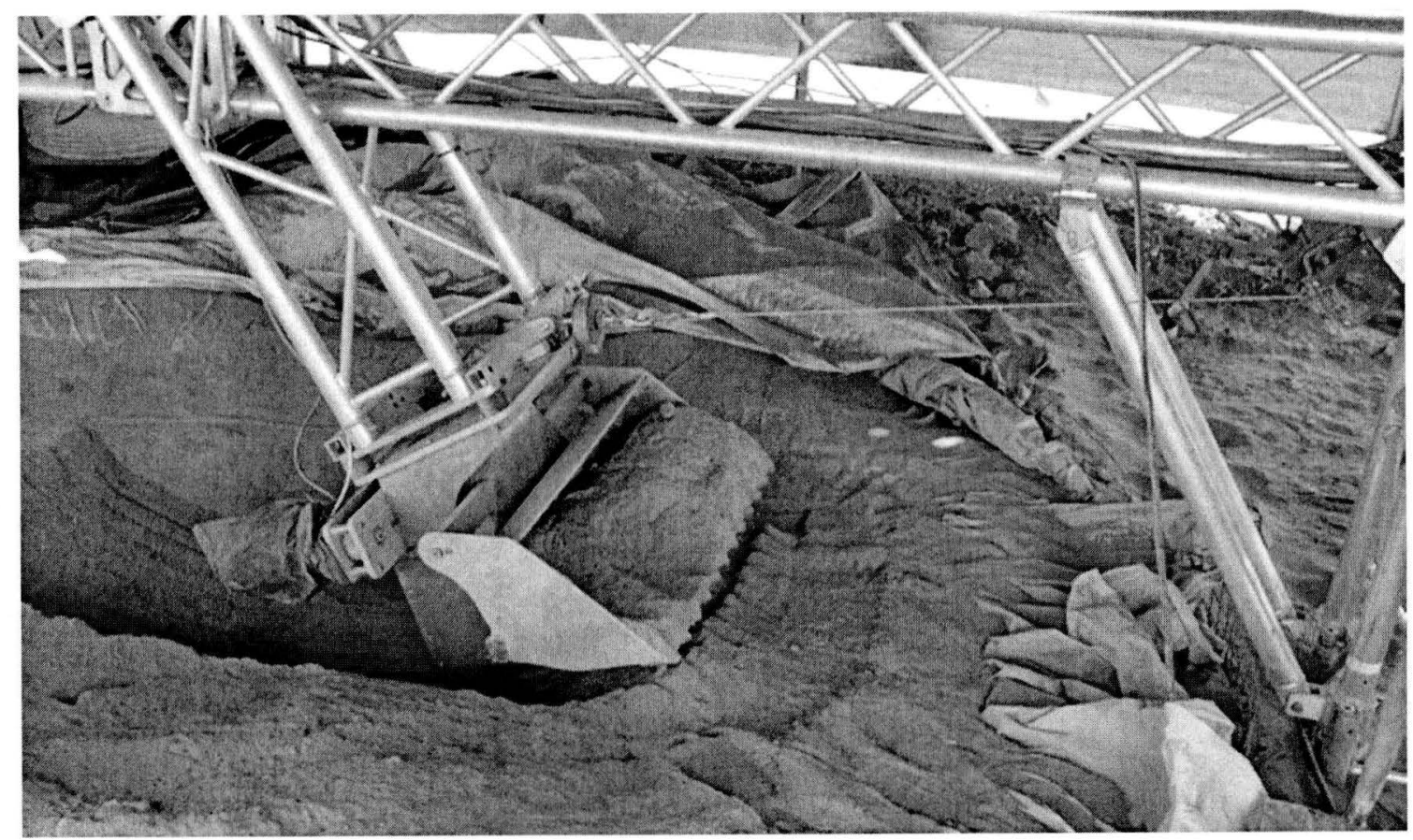

Figure 5 - VIPER Excavating BP-1 


\section{Lunar Simulant}

The lunar regolith simulant used in this experiment was Black Point 1 (BP-1) which is a low-cost physical simulant which is available and affordable for large scale testing. Other common simulants such as JSC-1A are prohibitively expensive and unavailable on a large scale ( $>5$ tons). BP-1 is composed of amygdaloidal nepheline-bearing basalt from the Black Point lava flow in northern Arizona [Ref Marshall paper]. The material is a waste product of a washing process at the local aggregate quarry. Examination has shown that the particle size distribution and geotechnical properties of BP-1 are more lunar-like than JSC-1A and comparable to high fidelity simulants such as NU-LH-T and CHENOBI [ref lalia].

\section{Testing Process}

The density of BR-1 Regolith can vary over a wide range based on its level of compaction (from TBD grams $/ \mathrm{cm}$ to TBD grams $/ \mathrm{cm}$ ). To ensure consistent and repeatable results we followed a set procedure for performing the digs and resetting the hole. First we set the zero by aligning the bucket so that it was perpendicular to the dig plane and lowering the bucket until the teeth on the bucket just touched. All further dig depths are referenced off of this zero. The bucket was then backed off, the depth set to 2" and a cut was made. At the end of each cut the bucket was manually cleared of regolith before the next cut was made. After the first cut of 2" was made, all additional cuts were made using a 1" depth of cut. The typical procedure was to cut from 2" down to a depth of 10 ", using the 1" depth of cut for each scoop, giving 8 data sets for one complete dig cycle. Each test was performed 5 times to ensure a statically significant data set.

Once the depth reached 10", the test was complete and the simulant could be reset for the next test. The procedure followed for this reset is as follows; the walls of the excavated hole were broken down and the regolith was manually raked back in the hole until it was slightly less than even with the surrounding grade. A large electric vibrating plate compactor was used to compact the regolith, going over the entire cut twice. The hole was then filled again until it was even with the surrounding grade and the large compactor run over the hole twice more. The hole was filled in once more to the surrounding grade and small plate compactors used for final finishing of the regolith.

Bulk density measurements were then taken in the compaction zone after a regolith reset, generally once in the morning and once in the afternoon or after any change in conditions which might affect the soil physical properties. The bulk density measurements were performed as follows: A 2.6 inch inner diameter ring, 4 inches long was driven into the regolith to a given depth. The regolith around the ring was excavated using a hand trowel. After a thin sheet of metal was slid underneath the ring, it was lifted out and the sample was placed in a bag and weighed.

\section{Test Variables}

Three variables were considered for this experiment: Excavation Speed, Frequency of Percussion, and Impact Energy (See Table 1). Excavation Speed was varied by controlling the voltage on the excavation winch through a variable transformer. Three voltages settings on a $110 \mathrm{VAC}$ circuit were used: $40 \%, 70 \%$, and $100 \%$. Because this was an open-loop control, the data were post-processed to measure the actual speed during the test and the results averaged. Frequency of percussion was varied by controlling the speed of the motor at the input of the percussive mechanism. The motor was outfitted with an incremental encoder and a commercial servo motor controller closed the loop on velocity to ensure constant velocity. The Impact Energy was varied by changing out springs on the percussion mechanism and the value was idealized to be the potential energy at the top of the ramp on the barrel cam (location of maximum spring compression).

Table 1 - Experimental Variables

\begin{tabular}{|c|c|c|}
\hline Variables & Units & Values Tested \\
\hline Excavation Speed & $\mathrm{cm} / \mathrm{s}$ & $3.8,8.8,11.7$ \\
\hline Frequency of Percussion & beats/minute (BPM) & $0,250,700$ \\
\hline Impact Energy & Joules & $13.6,19.3,30.5$ \\
\hline
\end{tabular}

\section{Testing Limitations}

There were several observations that were noticed during the months of testing. Some of these are related to testing outside. Testing outside presented several problems such as the wind blowing the small fines of the BP-1 simulant away. This was noticed during the last weeks of testing where the amount of dust generation was less compared to the beginning of testing. The test bin needed to be dug out and filled 3 separate times with dry BP-1 due to heavy rain saturating the regolith with water. This testing was all done in the spring and early summer where the temperatures reach into the upper $90 \mathrm{~s}$. The humidity in Florida may also impact the soil but this was never investigated. In 
combination with the heat and having to wear hooded Tyvex suits, full face respirators, and gloves rotations had to be made by several people during the course of testing.

\section{RESUltS}

\section{Data Processing}

This experiment produced over 1100 data files, each representing one pass of the excavation bucket. Each data file recorded the time, angle of the arm, and load cell reading. For the purposes of this paper, only tests performed at a depth of 8 " below surface level will be investigated. At this depth the bucket fills up completely and with minimal surcharge. This depth also provides consistent data because of its location toward the middle of the testing sequence which avoids errors due to resetting differences and edge effects.

As mentioned earlier, the raw data from the load cell was scaled using measurements from the VTS and a statics balance to give the load at the bucket tip. To ensure that this conversion was implemented properly a calibration test was performed. A tensile dynamometer was rigged in-line with the bucket tip and then secured to the VTS base structure. The excavation winch was then used to pull on the arm. A short data file was recorded and the post processed load cell reading was compared to the actual dynamometer reading. The maximum error was $2.5 \%$

Because of the pivoting design of the VTS arm the mass of the VIPER percussive mechanism and bucket have an effect on the excavation loads at the bucket tip during a test. The further away the center of gravity of VIPER is pulled away from below the pivot of the arm the more gravity will impart a torque on the arm. For the first half of the excavation pass this torque is reducing the excavation load and for the second half it is increasing it. To more accurately reflect the true excavation loads a series of tests were performed to calibrate out the effect of the system mass. The arm was swept through its limits over a previously excavated hole and collected no soil in the bucket while the load cell recorded the forces. This ramping load is symmetric about the point where the CG of VIPER is directly below the pivot of the arm. This symmetric load was then added to the post processing code to add or subtract the appropriate value at any given angle of the arm.

\section{Moving Average}

Upon inspection of the data from percussive tests, it was apparent that the percussion frequency emerges in the load cell data. As seen in Figure 6 the excavation load rises and falls at the same frequency as the percussion. This is assumed to be the result of the hammer hitting the anvil on the bucket and driving the arm quickly forward, reducing the load on the excavation winch. The winch then continues to retract and eventually catches up to the arm and resumes full load. It is unclear how the dynamic forces of the winch cable quickly loading and unloading may skew the data. This effect is only seen in the percussive tests, as the nonpercussive tests have smooth trends. In order to better compare the trends in the load data a moving average was applied to the percussive test data to remove the effect of the percussive frequency. The period of the moving average was based upon the frequency of percussion and the sampling rate. A minimum period was calculated that would remove only the high frequency effects of the percussion and not smooth out the overall trends in the data. 


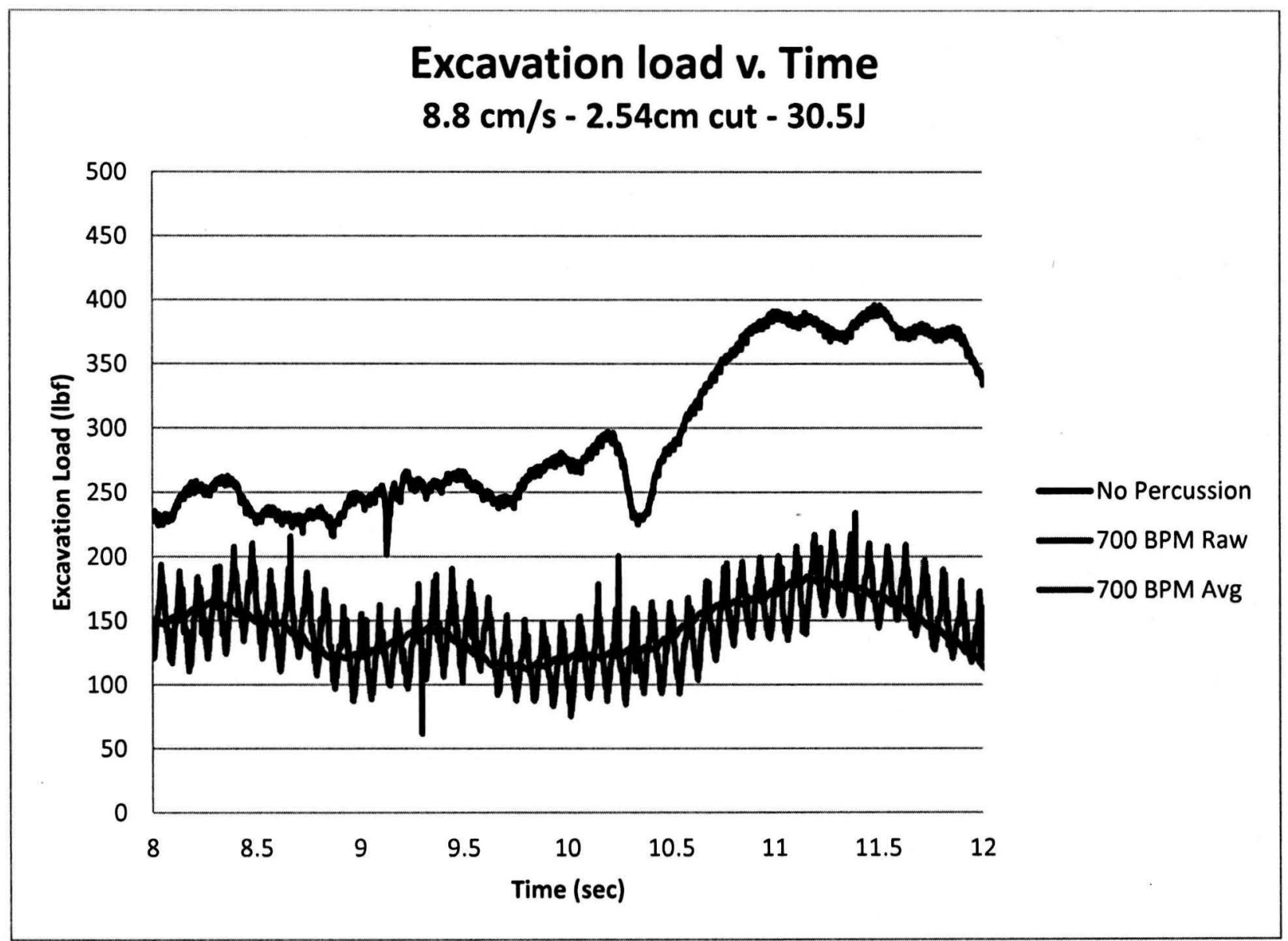

Figure 6 - Graph showing typical raw data with frequency and moving average

\section{Average Maximum Excavation Load}

In order to determine the excavation load reduction in the various test conditions, the moving average method described previously was applied to all of the percussive data sets and then the maximum load for each test was recorded. For each test condition there were approximately 5 trials. The maximum loads for each test condition were then averaged. This resulted in a comparison of the average maximum load for every percussive test with same for the non-percussive tests (see table 2).

\section{Trends in load reduction}

Graphs were also made to investigate the trends in excavation load as the three test variables (percussive frequency, energy, and speed) were increased. With these trends we can also compare this work with the experiment completed by Honeybee Robotics.

The first graph (see figure 7) shows the excavation load as the percussive frequency is increased while the series lines are constant excavation speeds. With all series, the load was reduced from no percussion to 250 BPM. From 250 to 700 however the load was relatively flat and even increased slightly in some cases. This trend differs from that seen by Honeybee Robotics which saw the exaction load consistently decrease with increasing percussive excavation into frequencies in the $1250 \mathrm{BPM}$ range. The $11.7 \mathrm{~cm} / \mathrm{s}$ series non percussive test falls below the slower $8.8 \mathrm{~cm} / \mathrm{s}$ non-percussive test which is somewhat unexpected.

The second graph (see figure 8) shows the excavation load as the impact energy is increased while the series lines are again constant excavation speeds. As the impact energy is increased the load is generally decreased however from the $19.3 \mathrm{~J}$ to $30.5 \mathrm{~J}$ tests the trends appear to level off. Once again the $11.7 \mathrm{~cm} / \mathrm{s}$ non percussive test shows a lower load than the slower $8.8 \mathrm{~cm} / \mathrm{s}$ test.

The third graph (see figure 9) shows the excavation load as the speed of excavation is increased while the series lines are constant impact energies. Increasing speed appears to increase the excavation load, however the effect becomes less pronounced in the higher speed and higher energy cases. This trend seems to be consistent with that seen in the Honeybee Robotics experiment. 
Table 2 - Average maximum load reduction data

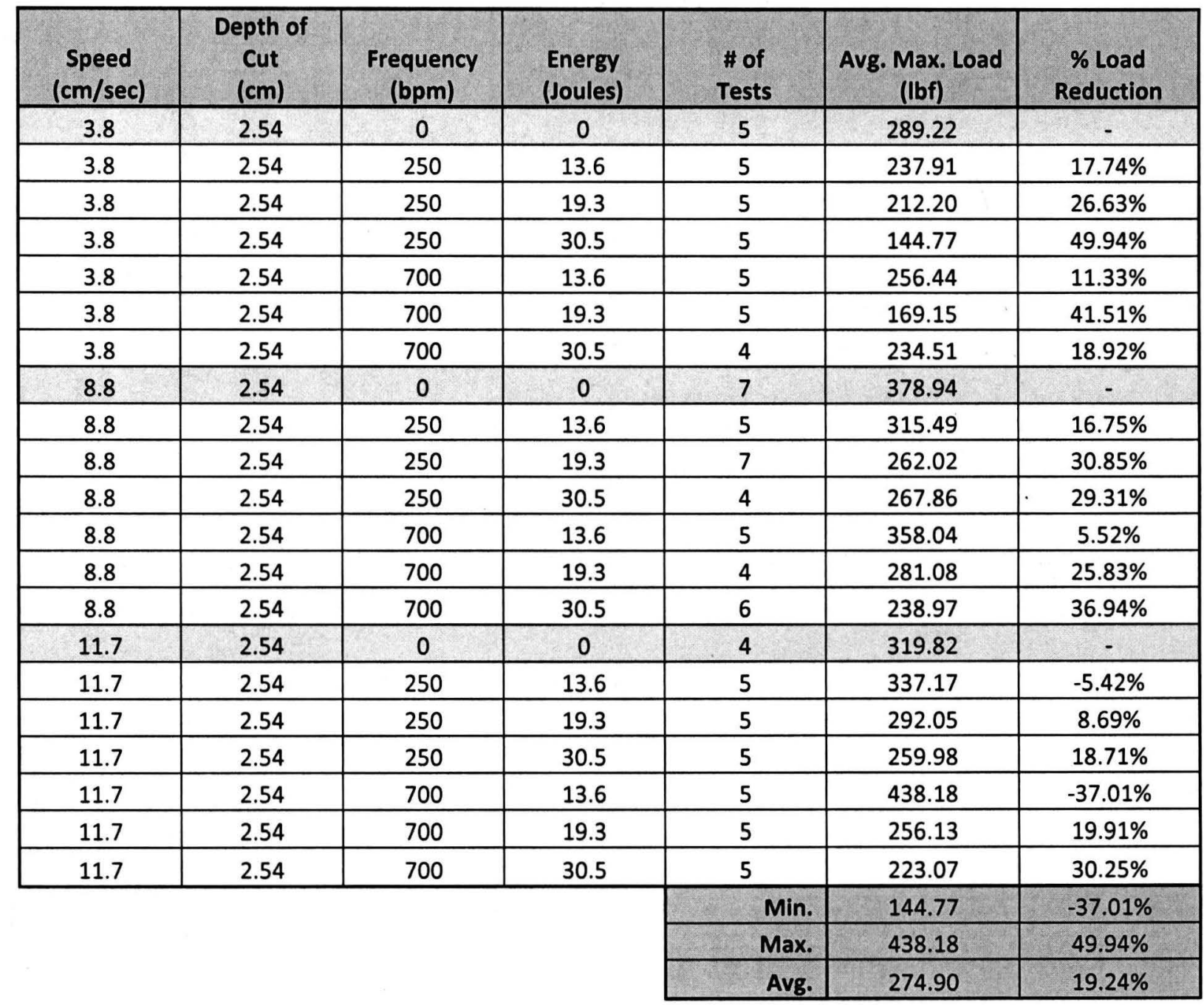

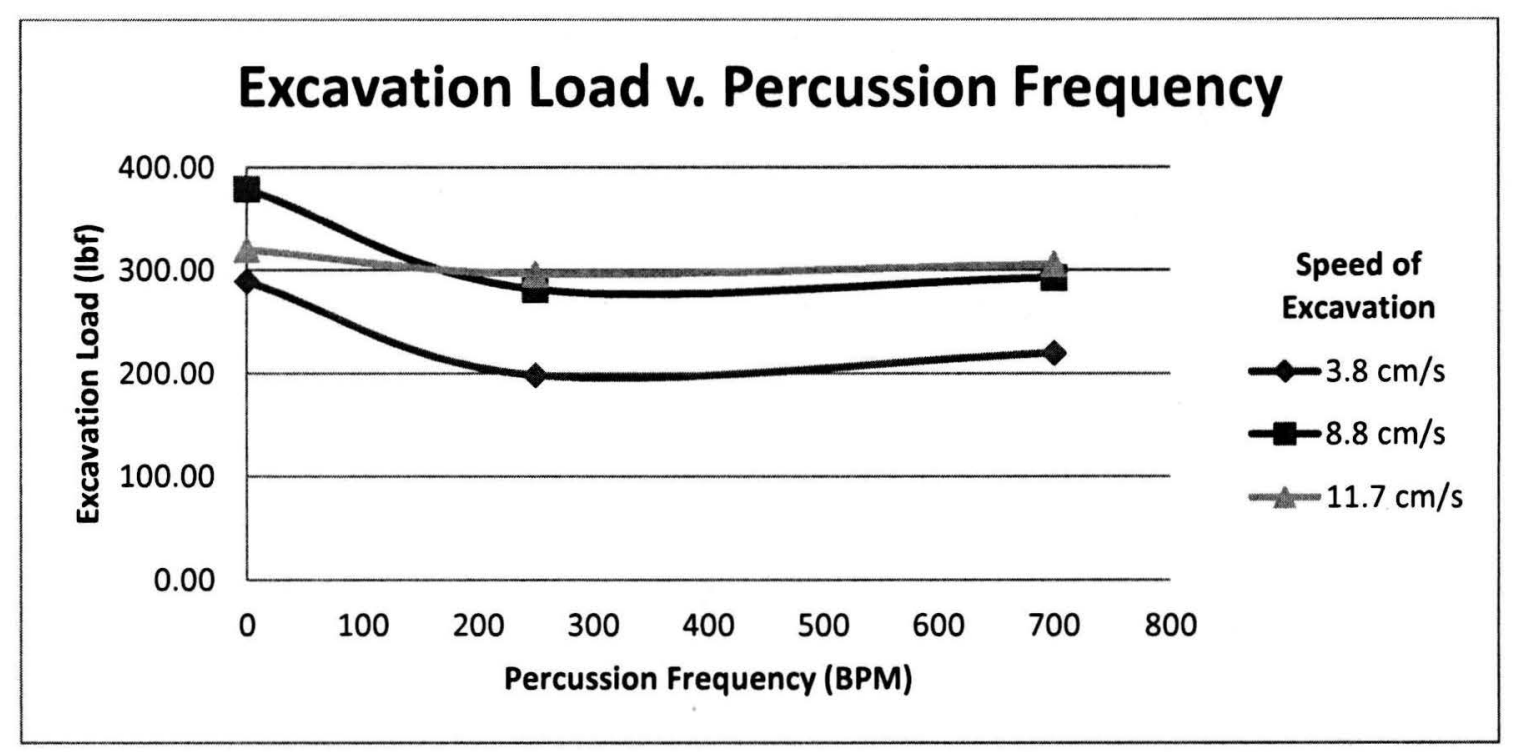

Figure 7 - Load trends with increasing percussive frequency 


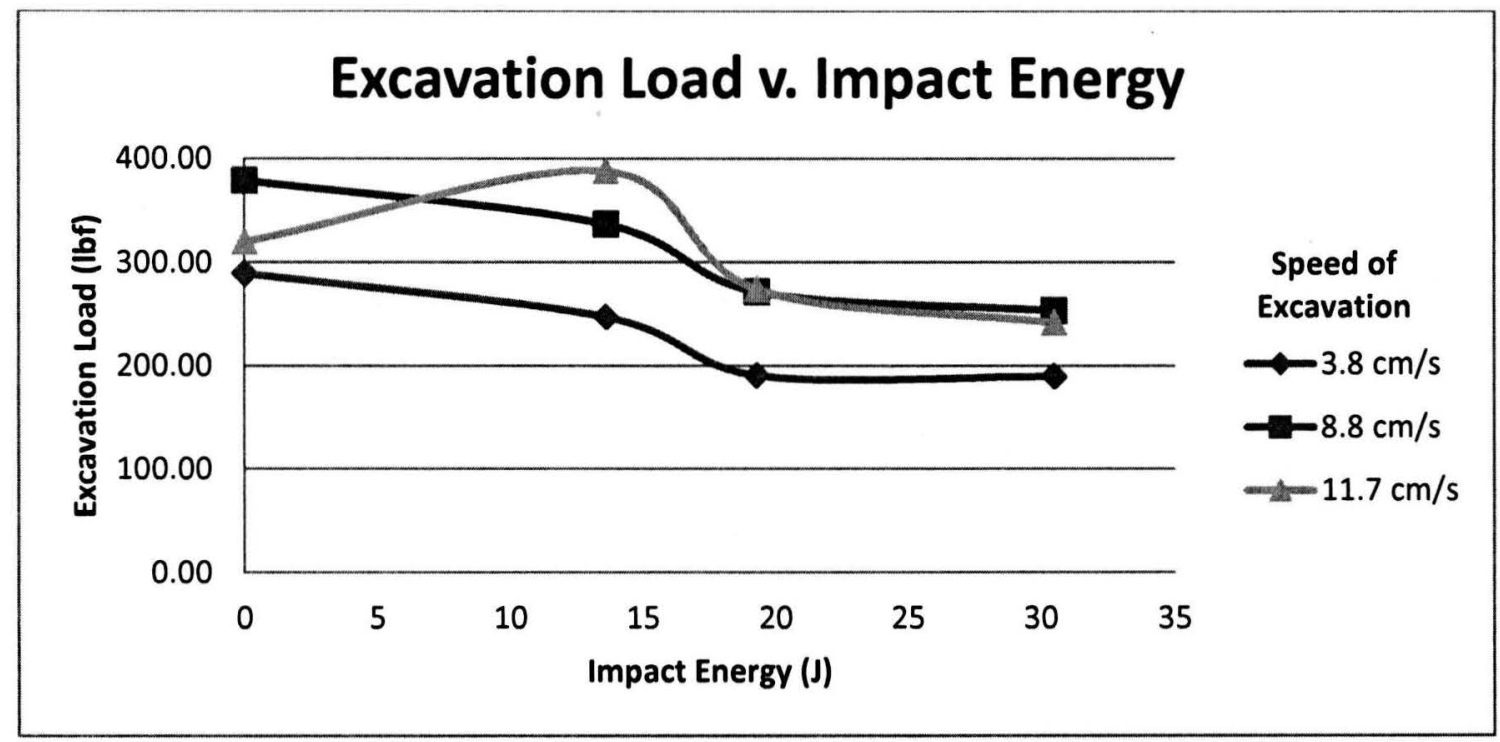

Figure 8 - Load trends with increasing impact energy

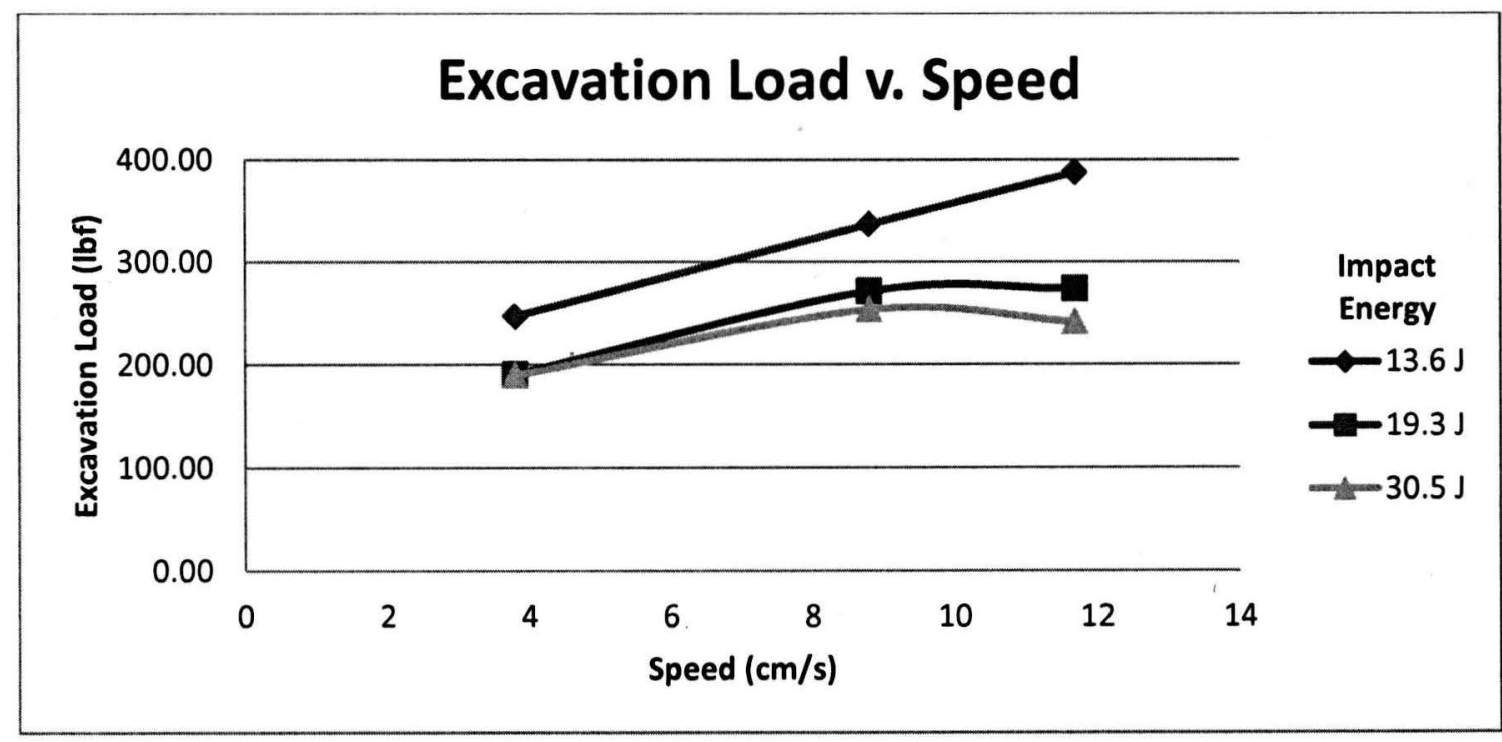

Figure 9 - Load trends with increasing excavation speed

\section{Conclusions}

\section{Variable Percussion Vector}

During testing it was found that the surcharge compacted in inside the bucket. From reviewing the results and watching videos from the Honeybee Robotics testing the surcharge remained fluidized and continued to roll over itself while the percussive mechanism was operating [Reference Honeybee paper]. There are many differences between the Honeybee Robotics setup, most notable scale and method of excavation (plow versus backhoe). Another difference that may play a role in the fluidization of the soil is the direction and method of energy transfer (rotational pivot versus linear slide). The Honeybee Robotics bucket impacted the bucket in the downward direction using a linear slide. With the scale of VIPER being larger it was a concern that applying the impact energy through the bucket would dissipate the energy being directed to the simulant. The VIPER impact occurred directly in line with the cutting edge, which forced the bucket to thrust forward through a pivot. It was assumed that this method would utilize the impact energy more efficiently. The low horizontal impact on VIPER is believed to contribute the compaction of the surcharge. The proposed cause for this compaction is the simulant being thrust into the back wall of the bucket with the horizontal impact. Further investigations into how the direction and method of energy transfer effect the surcharge will be performed in future testing. 


\section{Medium Scale Testing}

In 2013, the next project consists of a percussive backhoe style bucket, Badger, for the Centaur rover in combination with a robotic backhoe arm designed by Glenn Research Center. This percussive bucket will be $0.305 \mathrm{~m}$ (12 in) wide compared to the Honeybee Robotics Lunar Surveyor scoop at $0.0508 \mathrm{~m} \mathrm{(2} \mathrm{in)} \mathrm{and} \mathrm{the} \mathrm{VIPER} \mathrm{bucket} \mathrm{at} 0.762 \mathrm{~m} \mathrm{(30} \mathrm{in).}$ Assuming that leading difference between Honeybee Robotics' $70 \%$ reduction versus $50 \%$ on VIPER is mainly a scaling factor, Badger testing will include a larger spectrum sweep of impact force and frequency. Instead of the assumption that frequency does not scale with bucket width but impact force does. Also, more variations of direction and method of impact will be investigated.

\section{Icy Regolith Testing}

NASA KSC NE-S has an ongoing effort to produce quantities of icy lunar like material for the purpose of prototype lunar ice excavator development testing. Because evidence is mounting that permanently shadowed lunar craters contain water ice in the regolith at the lunar poles, $\mathrm{KSC}$ is conducting initial efforts to establish a frozen regolith excavation test capability in support of future lunar excavation tool development. Previous work done under a NASA SBIR identified a water content in lunar regolith of $8-10 \%$ produces the hardest frozen samples for excavation, so ongoing work in to reduce excavation energies in icy regolith is focused in this area.

\section{ACKNOWLEDGEMENTS}

The research reported in this manuscript was conducted by the NASA Surface Systems Office (NE-S) and Engineering Services Contract (ESC) at Kennedy Space Center.

\section{REFERENCES}

[1] (Luth, Harold J. and Wismer, Robert D. " Performance of Plane Soil Cutting Blades in Sand." Transactions of the ASAE. Vol. 14, pp 255 262, 1971.).

[2] http://usrp.usra.edu/technicalPapers/kennedy/WhitakerDec 08.pdf

[3] Colaprete, A., Schultz, P., Heldmann, J., Wooden, D., Shirley, M., Ennico, K., ... \& Sollitt, L. (2010). Detection of water in the LCROSS ejecta plume. Science, 330(6003), 463-468.

[4] Klosky, J. L., Sture, S., Ko, H. Y., \& Barnes, F. (1996, June). Vibratory excavation and anchoring tools for the lunar surface. ASCE.

[5] Mueller, R. P., \& Van Susante, P. J. (2011). A Review of Extra-Terrestrial Mining Robot Concepts. In Earth and Space 2012@ struction, and Operations in Challenging Environments (pp. 295-314). ASCE.
[6] Boles, ., Scott, W., and Connolly, . (1997). "Excavation Forces in Reduced Gravity Environment." J. Aerosp. Eng., 10(2), 99-103.

[7] Mueller, R. P. (2006). Surface Support Systems for CoOperative and Integrated Human/Robotic Lunar Exploration, IAC-06-A5.2.09, 57th International Astronautical Congress (IAC), October 2-6 2006, Valencia - Spain.

[8] Mueller, R., P., and King, R. H., (2008). "Trade study of excavation tools and equipment for lunar outpost development and ISRU." AIP Conf. Proc., 969, 237-244.

[9] Schmitt, J. (1991). Lunar sourcebook: A user's guide to the Moon. G. Heiken, D. Vaniman, \& B. M. French (Eds.). Cambridge University Press.

[10] Smith, P. H., Tamppari, L. K., Arvidson, R. E., Bass, D., Blaney, D., Boynton, W. V., ... \& Zent, A. P. (2009). $\mathrm{H} 2 \mathrm{O}$ at the Phoenix landing site. Science, 325(5936), 58-61.

[11] Stein Sture, J. Ledlie Klosky, Hon-Yim Ko, and Frank Barnes (1996). Vibratory Excavation and Anchoring Tools for the Lunar Surface, Proceedings of the Fifth International Conference on Space ' 96 held in Albuquerque, New Mexico, June 1-6, 1996 New York, NY: ASCE, 978-07844-0177-4 or 0-7844-0177-2, 1996, 1365 pp., 2 vols by Stewart W. Johnson, (editor), (Johnson \& Assoc., Albuquerque, NM)

[12] Skonieczny, K., \& Wettergreen, D. S. (2010). Parameters governing regolith site work by small robots.

[13] Wilcox, B. H., Litwin, T., Biesiadecki, J., Matthews, J., Heverly, M., Morrison, J., ... \& Cooper, B. (2007). ATHLETE: A cargo handling and manipulation robot for the moon. Journal of Field Robotics, 24(5), 421-434.

[14] Zacny, K., Mueller, R. P., Craft, J., Wilson, J., Hedlund, M., \& Cohen, J. (2010, March). Five-Step Parametric Prediction and Optimization Tool for Lunar Surface Systems Excavation Tasks. In Earth and Space 2010@, Engineering, Science, Construction, and Operations in Challenging Environments. ASCE. 
Biographies

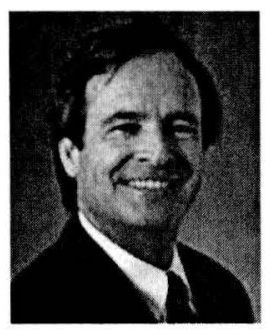

Robert Mueller received a B.S. in Mechanical Engineering from the University of Miami, Coral Gables, Florida, in 1988. In addition he has been awarded a Master of Space Systems Engineering from the Technical University of Delft, Netherlands and a Master of Business Administration, Florida Institute of Technology, Melbourne, Florida. He has 24 years of NASA mechanical engineering, robotics and management experience in aerospace operations, design and research \& technology development. Currently he is a senior aerospace technologist in the KSC Surface Systems Office and he is the NASA agency Lunar Destination Co-Lead for the Human Spaceflight Architecture Team (HAT). He has served as the Chief of the Surface Systems Office at $K S C$ and is actively engaged in technology development for NASA programs.

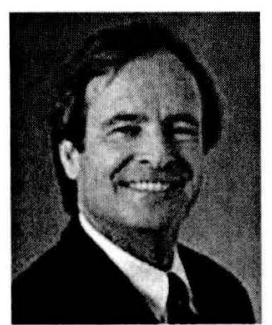

Thomas Lippitt is a graduate of the Georgia Institute of Technology. He worked for a petroleum pipeline company in Tulsa before coming to the Kennedy Space Center as a propellant systems design engineer with a specialty in high pressure and cryogenic systems. Additional duties at NASA include working in the Advanced Systems Development Laboratory performing implementation of automated systems and intelligent software. Tom is currently assigned in the KSC Surface System Office, developing technologies for mining on the moon and Mars.

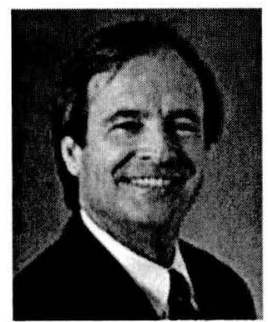

Jonathan Smith received a B.S. in Mechanical Engineering from The University of North Florida in 2009. He has been with Kennedy Space Center for 3 years. Currently he is working in the KSC Surface System Office, NE-S. Prior to NE-S he worked in the KSC Institutional Engineering Safety division where he led teams to define hazardous operations and implemented mitigating controls for cryogenics as well as many other tests and operations. Currently within NE-S he is working on percussive excavation technology to reduce excavation forces for low mass excavators in reduced gravity. He also is on a design team that is developing zero net reaction force excavating technology for micro excavators in reduced and micro gravity.

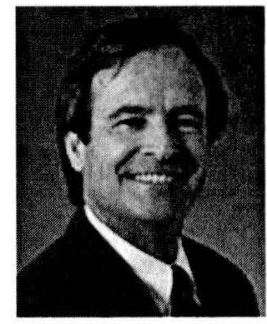

Andrew Nick received a B.S. in Mechanical Engineering from The University of North Florida in 2009. He has been with Kennedy Space Center for 3 years. Currently he is working in the KSC Surface System Office, NE-S. Prior to NE-S he worked in the KSC Institutional Engineering Safety division where he led teams to define hazardous operations and implemented mitigating controls for cryogenics as well as many other tests and operations. Currently within NE-S he is working on percussive excavation technology to reduce excavation forces for low mass excavators in reduced gravity. He also is on a design team that is developing zero net reaction force excavating technology for micro excavators in reduced and micro gravity.

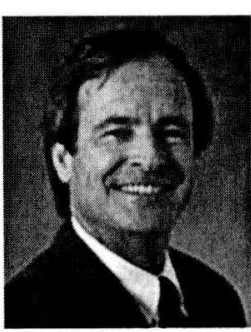

Jason Schuler received a B.S. in Mechanical Engineering from Florida Institute of Technology in 2007. He has been a contractor for NASA at the Kennedy Space Center for 5 years. Jason supports the NE-S office at KSC developing robotic technology and excavation tools for the moon and Mars. He has designed hardware for the SEV and ATHLETE rovers at JSC and JPL. On his personal time Jason is also heavily involved with FIRST robotics and educational outreach. 